\title{
Special issue in the honor of Daniel McFadden: introduction
}

\author{
André de Palma ${ }^{1} \cdot$ Nathalie Picard $^{2}$ (D) \\ Moshe Ben-Akiva ${ }^{3}$
}

Published online: 20 February 2018

(C) Springer Science+Business Media, LLC, part of Springer Nature 2017

\section{A tribute to Daniel McFadden}

Daniel McFadden received a Doctor Honoris Causa diploma from the University of Cergy-Pontoise, in December 2015. Many of his friends and researchers in the field of discrete choice analysis attended the Honoris Causa Ceremony and expressed their appreciation. A workshop on advances in discrete choice models took place at the University of Cergy-Pontoise during the following day.

Nathalie Picard

nathalie.picard@u-cergy.fr

1 University Paris-Saclay, Cachan, France

2 Universite de Cergy-Pontoise, Cergy, France

3 Massachusetts Institute of Technology, Cambridge, Massachusetts, USA 


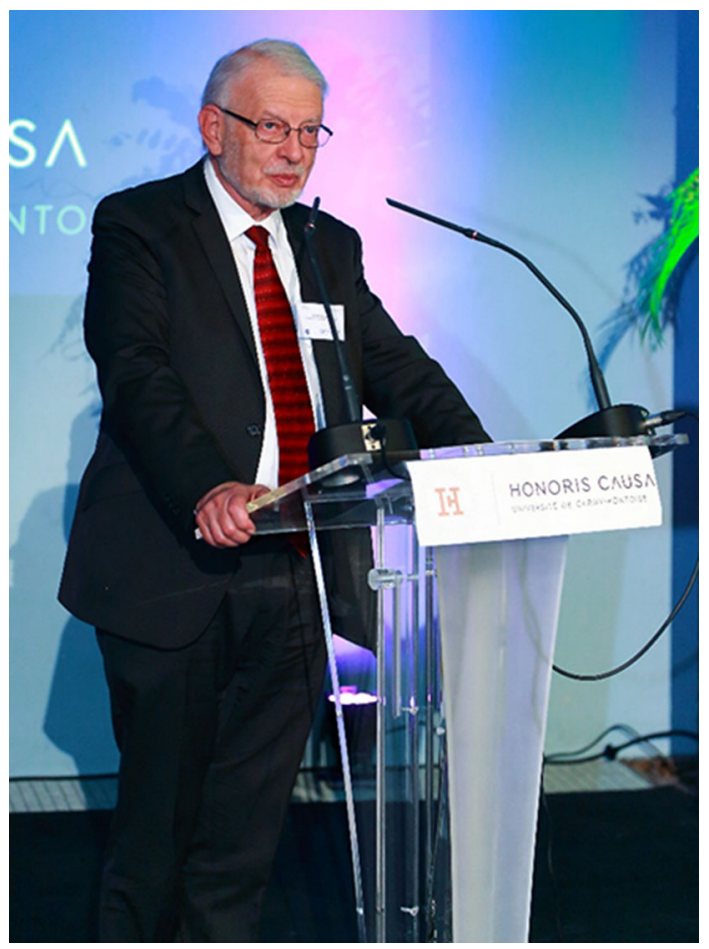

Daniel McFadden, at the University of Cergy-Pontoise, December 2015.

In this introductory note, we summarize the presentation of Dan's achievements during the Honoris Causa ceremony. Then, we introduce the papers that were presented at the workshop and included in this special issue.

Dan has previously visited the University of Cergy-Pontoise on several occasions, in particular to work on optimization problems in large-scale systems and a variety of other topics, including the economics of the family. Some of these topics have been discussed in a previous workshop and published in Ben-Akiva et al. 2012. Dan was also a member of the committee of Nathalie Picard's habilitation à diriger des recherches.

Dan's achievements are distinguished by his multi-disciplinary range. His research has brilliantly integrated the disciplines of economic theory, econometrics, optimization, behavioral science, psychology and psychophysics.

A few highlights of Dan's career:

- Dan's academic studies began in physics.

- Dan's economics career was spent at the following universities: University of Minnesota, MIT, University of California - Berkeley and the University of Southern California.

- Dan pioneered the area of microeconomics by advancing choice modeling, including Logit, Nested Logit, Logit Mixture, and hybrid choice models. Dan has developed, as did the physicist Werner Heisenberg, a new reasoning on things 
that can be observed and also on things that cannot be observed, but can, nevertheless, be modelled.

- Dan received the Nobel Prize in Economics in 2000 "for his development of theory and methods for analyzing discrete choice". His contributions to discrete choice also had major impacts on transportation science, industrial organization, regional science, finance, demography, social sciences, and marketing.

- Dan's research gave us new econometric, computational and operational dimensions that made discrete choice models widely used by practitioners, policy makers and professors.

- Dan published his research in various fields including Mathematical Economics, Transportation, Production theory, Environmental Economics and Health Economics, to name a few. He has published seminal papers that are highly cited.

There is another, perhaps lesser known, aspect of Dan's career and personal life that merits mention. In the 2006 issue of the Journal of Wine economics, Dan writes about wine and e-commerce (McFadden 2006). In his Nobel lecture, he also used wine to illustrate cognitive biases. Dan's friends know that Dan is not only a true wine connoisseur, but also has been a producer of top quality wines (even by the standards of French economists).

\section{Paper contributions}

The papers from the Advances in Discrete Choice workshop, in the three themes of: behavioral models, econometric models and industrial organization, are summarized below.

\subsection{Behavioral models}

This section contains three papers.

The first paper, Mobility decisions within couple, by Nathalie Picard (University of Cergy-Pontoise), Sophie Dantan and André de Palma (ENS Paris-Saclay) deals with discrete decisions involving several decision makers, a topic which has attracted Dan's attention since early 2000 (see Ben-Akiva et al. 2012). More specifically, the paper addresses the questions on how to extend individual choice models to a group of decision makers that have to make a single decision.

This extension of standard RUM (see Ben-Akiva and Lerman 1985) is very much in line with the joint decision which takes place in the context of the share of goods or services in collaborative consumption. In the context of this article, man and woman in the same household decide upon car ownership and mode choice (together with another important decision: who is the driver). The paper shows that omitting the collective aspects of the decision can bias considerably the estimations of individual preference parameters, and in particular the estimation of the value of time.

The second paper, Revisiting Consistency with Random Utility Maximization: Theory and implications for practical work, by Stephane Hess, Andrew Daly and Richard 
Batley (Institute for Transport Studies and Choice Modelling Centre), updates the historical perspective of discrete choice theory in McFadden's (2000).

The maximization of random utility has become a paradigm to describe probabilistic decision. According to the Thurstone law, an individual selects the alternative which maximizes his conditional utility. This model is consistent with the standard representative consumer approach, if the utility has a Gorman form (linear in income). In this case, ARUM (Additive Random Utility Models) with linear additive income are consistent with the standard axioms of consumer theory. For example, transitivity is satisfied, provided that the random terms are individual specific. The rationalization of discrete choice models remains partially an open issue. However, no author questions their relevance to study consumers' or agents' behavior. That is, their descriptive dimension remains unquestioned, while their normative dimension remains sometimes debatable.

The authors discuss random utility models, and point out the fact that several underlying behavioral assumptions, which have been observed and widely described in the literature, are not taken into account in the standard framework of discrete choice models.

The list includes:

Anchoring. Accordingly, previous choices may influence subsequent ones. Information acquisition, learning and social pressure can practically explain anchoring, but it still contains some elements of irrationality.

Zero cost/price effects. Zero cost plays some special role. Such behavioral rules needs to be inserted in RUM (Random Utility Models) .

Status quo. The idea is that changes are costly. This has to do with transaction costs, habit formation and inertia. Pieces have been modelled in RUM, but the topic remains challenging.

Mental accounting. Recently Richard Thaler was awarded the Nobel prize in Economics for his work on loss aversion and mental accounting. Mental accounting can be modelled by considering specific projects with their own benefits and costs not to be viewed as comprehensive objectives. Loss aversion corresponds to a discontinuity in the utility function or, more often, in the first derivative of the utility function.

Elimination by Aspect. A first EBA model was introduced by Amos Tversky the loyal coauthor of Daniel Kahneman, who received a Nobel prize for his contributions in behavioral finance.

The authors also mention other "anomalies": Lexicography, extreme sensitivity, reference dependence, loss aversion, decoy, framing, regret, and complexity. The paper addresses two challenges: how to model such anomalies, and how to be sure that RUM provides a good description of observed behavior. How such extended ARUM can be used to perform predictions, and to analyses the welfare dimension of individual choices remains an open question.

In the third paper, More on Random Utility Models with bounded ambiguity, Charles Manski (Northwestern University) develops a model that accounts for ambiguity:

RUM models are probabilistic choice models. There is no consensus as far as the origin of this probabilistic description. According to some authors, including according to early work of Manski, the probabilistic aspects are due to unobservable characteristic 
related to the alternatives or to the individual. According to some other authors, such as Luce and Tversky, the behavior of the decision maker is intrinsically probabilistic.

Here, Charles Manski studies some obvious aspects that are seldom modelled. Usually, the decision maker acts with a partial knowledge of the utilities. More realistically, the decision maker is facing uncertainty in the sense that $\mathrm{s} /$ he bases her/his decisions not on the actual distribution of attributes but on their subjectively perceived values. This information may be partially observable by the modeler, who has to infer the set of plausible subjective distributions. The failure to take this process into account may lead to severe misspecifications. A tractable example is constructed in the case of binary choice models. The situation where the decision maker has more than a single subjective distribution on the state of nature is referred to as behavioral ambiguity in Gilboa and Schmeidler (1989).

This paper is related to the paper of Walker et al. in this issue, which describes the decision-specific prior values to the parameters, to optimize an experimental design.

\subsection{Estimation techniques}

This section contains two papers.

The first paper, D-Efficient or Defficient, by Joan Walker, Yanqiao Wang (Berkeley), Mikkel Thorhauge (Technical University Denmark) and Moshe Ben-Akiva (MIT) is a review of experimental design for stated preferences studies.

There is a growing literature on the vulnerability of efficient experimental designs. Efficient design attempts to minimize the generalized variance or the standard errors of the parameters to be estimated (or maximizes the determinant of the information matrix).

The paper mainly focuses on the impact of mis-specification on efficiency and is complementary to the current literature which covers parameter bias.

Efficient designs assume that reliable prior ideas concerning the magnitude of the parameters to be estimated are available. An alternative is to perform a two-step procedure. In the first step, a small quantity of data are collected based on an uninformative prior. In the second stage, the initial estimates are used to create an efficient design. It is shown that the experimental design is effective if the initial values of the parameter estimated are near the true value of these parameters, but not if these initial estimates are outside the true range of parameters.

The second paper, A new Mixed MNP Model Accommodating a variety of Dependent Non-Normal Coefficient Distributions, by Chandra Bhat and Patricia Lavieri (The University of Texas at Austin) proposes a general copula approach to accommodate non-normal continuous mixing distributions in multinomial probit (MNP) models, relying on a new hybrid estimation technique combining maximum simulated likelihood and maximum approximate composite marginal likelihood (MACML) inference techniques. It is in the line of McFadden and Train (2000) observations that the logit mixture model is capable of approximating any random utility maximization model. This paper offers an interesting trade-off between tractability of computations and robustness of the method for general distributions of random coefficients. 
The effectiveness of the inference approach is demonstrated through simulation exercises as well as an empirical application.

\subsection{Industrial organization}

The last paper, Modelling purchases of new cars: an analysis of the 2014 French market, by Anna Fernandez-Antolin, Michel Bierlaire (EPFL, Ecole Polytechnique Fédérale de Lausanne) and Matthieu de Lapparent (School of Business, Management and Finance of Swiss University of Applied Sciences and Arts, Vaud) concerns an industrial organization dimension (for an introduction to IO and discrete choice models, see Anderson et al. 1992).

The paper addresses the analysis of the demand for new cars, included the intention to buy an electric car. The intention to buy a new car is measured either by the willingness to pay and or by the willingness to accept. The model used is the Cross-Nested logit, which has been developed initially by the transport community to address the problem of the correlation between similar alternative.

The proposed model allows to test different regulatory and price and incentive scenarios and to highlight the critical variable, which mostly explain consumer decision, such as the autonomy of the electric vehicles.

One of the contributions of this paper is an ability to impute the attributes of the unchosen alternatives based on the observed distribution of the alternatives.

Such analysis is useful, in particular, to study the market penetration of new alternatives, such as the electric cars and potentially the autonomous vehicles.

\section{References}

Anderson, S. P., de Palma, A., \& Thisse, J. F. (1992). Discrete choice theory of product differentiation. Cambridge: MIT Press.

Ben-Akiva, M. E., \& Lerman, S. R. (1985). Discrete choice analysis: theory and application to travel demand (Vol. 9). Cambridge: MIT press.

Ben-Akiva, M., de Palma, A., McFadden, D., Abou-Zeid, M., Chiappori, P.-A., de Lapparent, M., et al. (2012). Process and context in choice models. Marketing Letters, 23(2), 439-56.

Gilboa, I., \& Schmeidler, D. (1989). Maxmin expected utility with non-unique prior. Journal of mathematical economics, 18(2), 141-153.

McFadden, D. (2000). Disaggregate behavioral travel demand's RUM side—a 30 years retrospective. In D. A. Hensher (Ed.), Travel behaviour research (pp. 17-63). Amsterdam: Elsevier.

McFadden, 1. (2001). Economic Choices. The American Economic Review, 91(3), 351-378.

McFadden, D. (2006). Interstate wine shipments and e-commerce. Journal of Wine Economics, 1(01), 3-6.

McFadden, D., \& Train, K. (2000). Mixed MNL models for discrete response. Journal of Applied Econometrics, 15(5), 447-470.

Serres, M. (2012). Petite poucette (p. 68). Paris: Le pommier. 